\title{
Postpolymerization Synthesis of (Bis)Amide (Co)Polymers: Thermoresponsive Behavior and Self-association
}

Yicheng Zhu, Andrew B. Lowe, Peter J. Roth*

\author{
Yicheng Zhu, Prof. Andrew B. Lowe, Dr. Peter J. Roth \\ Centre for Advanced Macromolecular Design (CAMD), \\ School of Chemical Engineering, UNSW Australia, University of New South Wales, \\ Kensington, Sydney, NSW 2052, Australia \\ E-mail: P.Roth@unsw.edu.au
}

\begin{abstract}
A library of novel well-defined (bis)amide-based (co)polymers was prepared through postpolymerization modification of poly(pentafluorophenyl acrylate) with amines including ammonia and amide derivatives of amino acids. Products were characterized using a combination of NMR and FT-IR spectroscopies and size-exclusion chromatography; results conformed to the expected structures obtained through quantitative conversion. The series of (bis)amide (co)polymers displayed rich phase behavior in aqueous solution such as thermoreversible gelation at low temperature and high concentration while other samples displayed inverse temperature dependent solubility (lower critical solution temperature (LCST)-type) behavior. A hydrophobically modified polyacrylamide copolymer displayed upper critical solution temperature (UCST) behavior in aqueous solution. Significantly, driven by polymer-polymer hydrogen bonding, copolymers self-associated into highly ordered, regular structures of several tens to hundreds of micrometers in size. Morphologies included sheet-like, rod-like and honeycomb-like structures and depended strongly on the chemical composition of copolymers.
\end{abstract}




\section{Introduction}

Several polyacrylamide species comprised of $\mathrm{N}$-acryloyl derivatives of amino acid amides have recently become the focus of intense research in different fields of polymer science. Featuring two (or more) amide groups in each monomer segment, each acting as hydrogen bond donor and acceptor, the behavior of these hydrophilic polymers is primarily dictated by strong intra- and intermolecular polymer-polymer interactions. Depending on temperature these attractions can outweigh polymer-water interactions in aqueous solution. The simplest amino acid amide-based polyacrylamide, poly( $N$-acryloyl glycinamide) (pGlyAm), for example, exhibits thermally reversible protein-like gelation in water and physiological buffer solution at low temperature due to significant hydrogen bonding interactions.[1, 2] Moreover, pGlyAm, poly( $N$-acryloyl asparaginamide) (pAspAm), and poly $(N$-acryloyl glutaminamide) (pGlnAm) were recently reported by Ohnishi's group, [3-5] and others, [6, 7] to exhibit upper critical solution temperature (UCST) behavior in water-a rare phenomenon observed only for a few thermoresponsive (co)polymers.[8, 9] Research in Agarwal's group revealed that the behavior of pGlyAm depends strongly on the synthetic procedure used to produce this species, with UCST transitions occurring only if rigorous care is taken to avoid incorporation of any traces of ionic groups into the polymer.[10] With appropriate synthetic care however, pGlyAm exhibiting sharp reproducible UCST transitions is accessible.[11, 12] Similarly, hydrophobically modified polyacrylamido species have been shown to exhibit UCST transitions in water, likewise governed by inter-polymer hydrogen bonding between amide groups.[13]

Postpolymerization modification[14-18] is a versatile technique for the synthesis of functional (co)polymers and has lent itself perfectly to the investigation of structure-property relationships in thermoresponsive materials since libraries of homopolymers and statistical copolymers with identical average degrees of polymerization and controllable molar compositions can easily be prepared.[19-28] Of a wide variety of stimulus responsive 
(co)polymers prepared through postpolymerization modification techniques, only very few (co)polymer species exhibit aqueous UCST behavior. Examples include ureido-functional copolymers[29, 30] and zwitterionic polysulfobetaines.[31] Activated esters such as pentafluorophenyl (meth)acrylate (PFP(M)A) constitute a very versatile class of reactive scaffold.[18] Exhibiting highly selective reactivity toward primary and secondary amines, PFP(M)A polymers (pPFP(M)A) provide simple access to functional poly $(N$-alkyl (meth)acrylamide)s and poly( $N, N$-bisalkyl (meth)acrylamide)s including several wellinvestigated lower critical solution temperature (LCST)-type thermoresponsive species, e.g. $\operatorname{poly}(N$-isopropylacrylamide) (pNIPAM).[24]

In this study we investigated the modification of reactive pPFPA scaffolds as a route to (bis)amide functional polymeric species including hydrophobically modified polyacrylamides, pGlyAm, and several novel amino acid amide-derived (co)polymers. We took advantage of the postpolymerization concept to produce a range of homopolymers and statistical copolymers with an initial goal of probing aqueous solution behavior and identifying novel thermoresponsive (co)polymers. While UCST behavior was found for only one sample, several (co)polymers showed thermoreversible gelation or LCST transitions in water. Additionally, due to their strong hydrogen-bonding capacity, we found (co)polymers able to self-associate into tubular or sheet-like structures in the dry state, offering a novel approach to well-defined self-assembled morphologies. 


\section{Experimental Section}

\section{Instrumentation}

NMR spectroscopic measurements were performed on a Bruker Avance $300 \mathrm{MHz}$ instrument. Internal solvent signals were used as reference: $\delta\left(D M S O-d_{6}\right)=2.50 \mathrm{ppm}, \delta\left(\mathrm{D}_{2} \mathrm{O}\right)=4.79 \mathrm{ppm}$. Size exclusion chromatography (SEC) was performed on a Shimadzu system equipped with four $300 \times 7.8 \mathrm{~mm}^{2}$ linear phenogel columns $\left(10^{5}, 10^{4}, 10^{3}\right.$ and $\left.500 \AA\right)$ operating at a flow rate of $1 \mathrm{~mL} / \mathrm{min}$ using dimethylacetamide as eluent. The system was calibrated with a series of narrow molar mass distribution polystyrene standards with molar masses ranging from $0.58-1820 \mathrm{~kg} / \mathrm{mol}$.

Fourier transform infrared (FT-IR) spectroscopy was performed on a Bruker IFS 66/S instrument under attenuated total reflectance (ATR) and data was analyzed on OPUS software version 4.0 .

Dynamic light scattering (DLS) measurements were performed on a Malvern Zetasizer Nano $\mathrm{ZS}$ at a scattering angle of $173^{\circ}$ and were analyzed by Malvern Zetasizer Software version 6.20.

Turbidity measurements were performed on a Varian Cary 300 Scan spectrophotometer equipped with a Cary temperature controller and a Peltier heating element in quartz cuvettes of $10 \mathrm{~mm}$ path length at a wavelength of $520 \mathrm{~nm}$ with heating / cooling rates of $1{ }^{\circ} \mathrm{C} / \mathrm{min}$. Polymer concentrations were $5 \mathrm{~g} / \mathrm{L}$. For clear solutions the baseline was corrected to zero absorbance, $A$. Transmittance, $T=10^{-A}$, was plotted against temperature and cloud points were determined at $T=50 \%$.

Scanning electron microscopy (SEM) was conducted on a FEI Nova NanoSEM 230 using an Everhart-Thornley (ET) detector and an accelerating voltage of $5 \mathrm{kV}$. Samples were freezedried from aqueous solution (concentration $=2 \mathrm{~g} / \mathrm{L}$ ), carefully deposited on a sticky carbon film and plasma-coated with a thin layer of chromium. 


\section{Materials and Synthesis}

Amide derivatives of amino acids glycinamide $\mathrm{HCl}$ (1), L-alaninamide $\mathrm{HCl}$ (2), Lserinamide $\cdot \mathrm{HCl}(3)$, L- $\alpha$-aminobutyramide $\cdot \mathrm{HCl}(\mathbf{4})$, L-valinamide $\cdot \mathrm{HCl}(\mathbf{5})$, L-leucinamide $\cdot \mathrm{HCl}$ (6), $\beta$-alaninamide $\cdot \mathrm{HCl}(7)$, and the dipeptide glycylglycine (8), were purchased from ChemImpex International. All other reagents were purchased from Sigma-Aldrich and used as received. Pentafluorophenyl acrylate was prepared as published elsewhere.[31]

Poly(pentafluorophenyl acrylate), pPFPA, with two different molar masses was prepared by the RAFT process using benzylpropyltrithiocarbonate as chain transfer agent in analogy to a literature procedure.[31] pPFPA1, $M_{\mathrm{n} \text {,theor. }}($ from conversion $)=24.7 \mathrm{~kg} / \mathrm{mol}$., $D P_{\text {theor. }}=103$, $M_{\mathrm{n}, \mathrm{SEC}}=46.8 \mathrm{~kg} / \mathrm{mol}(\mathrm{PS}$ standard$), \bigoplus_{\mathrm{M}}=M_{\mathrm{w}, \mathrm{SEC}} M_{\mathrm{n}, \mathrm{SEC}}=1.35 ;$ pPFPA2, $M_{\mathrm{n}, \text { theor. }}$ (from conversion $)=9.5 \mathrm{~kg} / \mathrm{mol} ., D P_{\text {theor. }}=39, M_{\mathrm{n}, \mathrm{SEC}}=19.8 \mathrm{~kg} / \mathrm{mol}(\mathrm{PS}$ standard $), \bigoplus_{\mathrm{M}}=$ $M_{\mathrm{w}, \mathrm{SEC}} / M_{\mathrm{n}, \mathrm{SEC}}=1.40 .{ }^{19} \mathrm{~F} \mathrm{NMR}\left(290 \mathrm{MHz}, \mathrm{CDCl}_{3}\right), \delta / \mathrm{ppm}=-153.2(\mathrm{bm}, 2 \mathrm{~F}$, ortho $),-157.1$ (bs, $1 \mathrm{~F}$, para), -162.4 (bs, $2 \mathrm{~F}$, meta).

Postpolymerization Modification of pPFPA with Amines: General Procedure. pPFPA (166.6 mg, $0.7 \mathrm{mmol}$ of repeat units) was dissolved in anhydrous $\mathrm{N}, \mathrm{N}$-dimethylformamide (DMF, $2 \mathrm{~mL}$ ) and acrylamide (4 mg, $0.056 \mathrm{mmol}$ ) (to scavenge thiol groups generated by RAFT end group cleavage) was then added. In a separate vial an amine (see table 1) (1.75 mmol, 2.5 equiv.) was dissolved in DMF (1 mL). For amines used as hydrochloride salts, 1,8-diazabicycloundec-7-ene (DBU, $0.419 \mathrm{~mL}, 2.8 \mathrm{mmol}, 4$ equiv.) was added. The amine solution was then quickly added into the polymer solution and the mixture stirred at room temperature overnight. Copolymers were prepared by an analogous protocol using a mixture of two different amines (total amount $1.05 \mathrm{mmol}, 1.5$ equiv). For example, for a reaction containing a feed of $50 \%$ of alaninamide and $50 \%$ of glycinamide, 0.75 equiv of Lalaninamide $\cdot \mathrm{HCl}$ and 0.75 equiv of glycinamide $\cdot \mathrm{HCl}$ were mixed with $\mathrm{DBU}$ in $\mathrm{DMF}$, and then introduced into a solution containing 1 equiv. of PFPA groups. Product polymers were purified by dialysis against water in regenerated cellulose membranes with a molecular 
weight cut-off of $3500 \mathrm{~g} / \mathrm{mol}$ for 3 days with solvent changes 3 times a day, followed by freeze-drying. Yields ranged between 83 and $91 \%$. GlyAm (co)monomer units, ${ }^{1} \mathrm{H}$ NMR $\left(\mathrm{DMSO}_{-} \mathrm{d}_{6}, 300 \mathrm{MHz}\right), \delta / \mathrm{ppm}=3.65\left(-\mathrm{NHC}_{2}-\right)$. AlaAm (co)monomer units, ${ }^{1} \mathrm{H}$ NMR $\left(\mathrm{DMSO}_{-} \mathrm{d}_{6}, 300 \mathrm{MHz}\right), \delta / \mathrm{ppm}=4.21(-\mathrm{NHC} \underline{\mathrm{H}}<), 1.25\left(-\mathrm{C}_{3}\right)$. SerAm (co)monomer units, ${ }^{1} \mathrm{H}$ NMR (DMSO-d $\left.6,300 \mathrm{MHz}\right), \delta / \mathrm{ppm}=4.96,4.34\left(-\underline{\mathrm{C}}_{2} \mathrm{OH}\right), 3.66(-\mathrm{NHC} \underline{\mathrm{H}}<) . \quad$ AbuAm (co)monomer units, ${ }^{1} \mathrm{H}$ NMR (DMSO-d $\left.6,300 \mathrm{MHz}\right), \delta / \mathrm{ppm}=4.16(-\mathrm{NHC} \underline{\mathrm{H}}<), 1.58(-$ $\left.\mathrm{C}_{2} \mathrm{CH}_{3}\right), 0.88\left(-\mathrm{CH}_{2} \underline{\mathrm{CH}}_{3}\right)$. ValAm (co)monomer units, ${ }^{1} \mathrm{H}$ NMR (DMSO-d 6 , $300 \mathrm{MHz}$ ), $\delta / \mathrm{ppm}=4.10(-\mathrm{NHC} \underline{\mathrm{H}}<), 2.44\left(-\mathrm{C} \underline{\mathrm{H}}\left(\mathrm{CH}_{3}\right)_{2}\right), 0.87\left(-\mathrm{CH}\left(\mathrm{CH}_{3}\right)_{2}\right)$. LeuAm $(\mathrm{co})$ monomer units, ${ }^{1} \mathrm{H}$ NMR (DMSO-d $\left.6,300 \mathrm{MHz}\right), \delta / \mathrm{ppm}=4.16(-\mathrm{NHC} \underline{\mathrm{H}}<), 2.43\left(-\mathrm{CH}_{2} \mathrm{C} \underline{\mathrm{H}}\left(\mathrm{CH}_{3}\right)_{2}\right), 1.58(-$ $\left.\mathrm{C}_{2} 2 \mathrm{CH}\left(\mathrm{CH}_{3}\right)_{2}\right), 0.88\left(-\mathrm{CH}_{2} \mathrm{CH}\left(\mathrm{C}_{3}\right)_{2}\right)$. $\beta$-AlaAm (co)monomer units, ${ }^{1} \mathrm{H}$ NMR (DMSO-d 6 , $300 \mathrm{MHz}), \delta / \mathrm{ppm}=3.56\left(-\mathrm{NHC}_{2} \mathrm{CH}_{2}-\right), 2.66\left(-\mathrm{NHCH}_{2} \underline{\mathrm{C}}_{2}-\right)$. GlyGlyAm (co)monomer units, ${ }^{1} \mathrm{H}$ NMR $\left(\mathrm{D}_{2} \mathrm{O}, 300 \mathrm{MHz}\right), \delta / \mathrm{ppm}=4.22\left(-\mathrm{NHC}_{2} \mathrm{COOH}\right), 3.63\left(-\mathrm{NHC}_{2} \mathrm{CONH}-\right)$. BzAm (co)monomer units, ${ }^{1} \mathrm{H}$ NMR $\left(\right.$ DMSO-d $\left._{6}, 300 \mathrm{MHz}\right), \delta / \mathrm{ppm}=7.33,7.26(\mathrm{~m},-$ $\mathrm{CH}_{2} \mathrm{C}_{6} \underline{\mathrm{H}}_{5}$ ), 4.29 (- $\left.\underline{\mathrm{CH}}_{2} \mathrm{C}_{6} \mathrm{H}_{5}\right)$. HexAm (co)monomer units, ${ }^{1} \mathrm{H}$ NMR (DMSO-d 6 , $300 \mathrm{MHz}$ ), $\delta / \mathrm{ppm}=3.01\left(-\mathrm{NHCH}_{2} \mathrm{CH}_{2} \mathrm{CH}_{2} \mathrm{CH}_{2} \mathrm{CH}_{2} \mathrm{CH}_{3}\right), 1.40\left(-\mathrm{NHCH}_{2} \mathrm{C}_{2} \mathrm{CH}_{2} \mathrm{CH}_{2} \mathrm{CH}_{2} \mathrm{CH}_{3}\right), 1.25(-$ $\left.\mathrm{NHCH}_{2} \mathrm{CH}_{2} \underline{\mathrm{C}}_{2} \mathrm{C}_{2} \mathrm{C}_{2} \underline{\mathrm{CH}}_{3}\right), 0.88\left(-\mathrm{NHCH}_{2} \mathrm{CH}_{2} \mathrm{CH}_{2} \mathrm{CH}_{2} \mathrm{CH}_{2} \mathrm{C}_{3}\right)$. DEGAm (co)monomer units, ${ }^{1} \mathrm{H}$ NMR (DMSO-d 6 , $\left.300 \mathrm{MHz}\right), \delta / \mathrm{ppm}=3.52,3.45\left(-\mathrm{NHCH}_{2} \underline{\mathrm{C}}_{2} \mathrm{OC}_{2} \underline{\mathrm{C}}_{2} \underline{\mathrm{OCH}}_{3}\right)$, 3.25 (- $\mathrm{NHC}_{2} \mathrm{CH}_{2} \mathrm{OCH}_{2} \mathrm{CH}_{2} \mathrm{OC}_{3}$ ). PentAm (co)monomer units, ${ }^{1} \mathrm{H}$ NMR (DMSO-d 6 , 300 $\mathrm{MHz}), \delta / \mathrm{ppm}=3.01\left(-\mathrm{NHC}_{2} \mathrm{CH}_{2} \mathrm{CH}_{2} \mathrm{CH}_{2} \mathrm{CH}_{3}\right), 1.40\left(-\mathrm{NHCH}_{2} \underline{\mathrm{CH}}_{2} \mathrm{CH}_{2} \mathrm{CH}_{2} \mathrm{CH}_{3}\right), 1.24(-$ $\left.\mathrm{NHCH}_{2} \mathrm{CH}_{2} \underline{\mathrm{C}}_{2} \mathrm{C}_{2} \mathrm{CH}_{3}\right), 0.87$ (- $\left.\mathrm{NHCH}_{2} \mathrm{CH}_{2} \mathrm{CH}_{2} \mathrm{CH}_{2} \mathrm{C}_{3}\right)$. IPAm (co)monomer units, ${ }^{1} \mathrm{H}$ NMR (DMSO-d 6 , $300 \mathrm{MHz}), \delta / \mathrm{ppm}=3.84\left(-\mathrm{NHC} \underline{\mathrm{H}}\left(\mathrm{CH}_{3}\right)_{2}\right), 1.04\left(-\mathrm{NHCH}\left(\mathrm{C}_{3}\right)_{2}\right)$. 


\section{Results and Discussion}

\section{Synthesis}

Postpolymerization modification of pPFPA precursors successfully yielded a library of welldefined amino acid amide-functional bisamide (co)polymers. Two reactive precursors, pPFPA1 $\left(M_{\mathrm{n}, \text { theor. }}=24.7 \mathrm{~kg} / \mathrm{mol}, \bigoplus_{\mathrm{M}}=1.35\right)$ and pPFPA2 $\left(M_{\mathrm{n}, \text { theor. }}=9.5 \mathrm{~kg} / \mathrm{mol}, \bigoplus_{\mathrm{M}}=1.40\right)$, prepared by the RAFT process,[32] were reacted in DMF with amide-functional amines and amine mixtures, producing bisamide homo- and copolymers, which were purified by dialysis against ultrapure water. The selection of amines comprised amide derivatives of the amino acids glycine (Gly), alanine (Ala), serine (Ser), valine (Val), and leucine (Leu), amino acid analogs $\beta$-alanine ( $\beta$-Ala) and 2-aminobutyric acid (Abu), the dipeptide glycylglycine (GlyGly), as well as common hydrophilic and hydrophobic amines (hexylamine, pentylamine, benzylamine, isopropylamine, and di(ethylene glycol) methyl ether (DEG) amine). Hydrophobically modified acrylamide copolymers were prepared by reaction of pPFPA with ammonia in combination with benzylamine. Chemical structures of all amines are given in Scheme 1; Table 1 gives an overview of the (co)polymers prepared. Aminolysis of activated PFP ester side groups can be expected to cleave the trithiocarbonate end groups, and is, in fact, the most common way for effecting RAFT end group modification.[33, 34] In order to prevent side reactions of the resulting thiol end groups, acrylamide was added as a thiol scavenger (Michael acceptor), which, featuring an amide moiety, produces end groups with high chemical similarity to the (bis)amide repeating groups, thus eliminating possible end groups effects on aqueous solution behavior (see Scheme 1).

Successful reaction was confirmed by a combination of FT-IR and NMR spectroscopies, as well as size exclusion chromatography (SEC). Representative ${ }^{1} \mathrm{H}$ NMR spectra of homo- and copolymers are shown in Figure 1A and the supporting information. Spectra showed signals conforming to the structures expected for quantitative reaction of the PFP functional groups. 
Molar compositions of copolymers were determined by comparison of the integrals of key resonances of each comonomer functionality and are listed in Table $1 .{ }^{19} \mathrm{~F}$ NMR spectroscopy of reaction mixtures before purification showed signals characteristic of free pentafluorophenol and no remaining PFP ester signals, confirming full reaction of the PFP ester groups, see supporting information. A comparison of FT-IR spectra of bisamide product (co)polymers with a spectrum of a pPFPA precursor, shown in Figure 1B, indicated the complete disappearance of characteristic bands associated with the precursor ( $\mathrm{PFP}$ ester $\mathrm{C}=\mathrm{O}$ stretching at $1780 \mathrm{~cm}^{-1}, \mathrm{C}-\mathrm{F}$ stretching at $1520 \mathrm{~cm}^{-1}$, and aromatic $\mathrm{C}=\mathrm{C}$ stretching-multiple weak bands from 1480-1440 $\mathrm{cm}^{-1}$ ) and the appearance of bands characteristic of secondary amides (N-H stretching at $3500-3250 \mathrm{~cm}^{-1}, \mathrm{C}=\mathrm{O}$ stretching (amide I band) at $1720-1600$ $\mathrm{cm}^{-1}, \mathrm{~N}-\mathrm{H}$ bending (amide II band) at $1580-1500 \mathrm{~cm}^{-1}$ ) during the reaction, likewise confirming complete modification. SEC (of species soluble in dimethylacetamide) revealed product (co)polymers to have molar mass distributions similar to the parent precursors with no evidence of crosslinking or polymer decomposition, see supporting information and Table 1. 


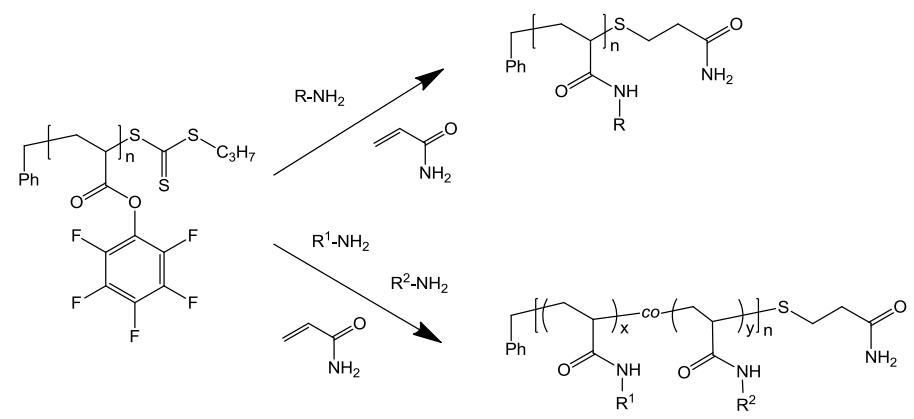

R-NH ${ }_{2}=$

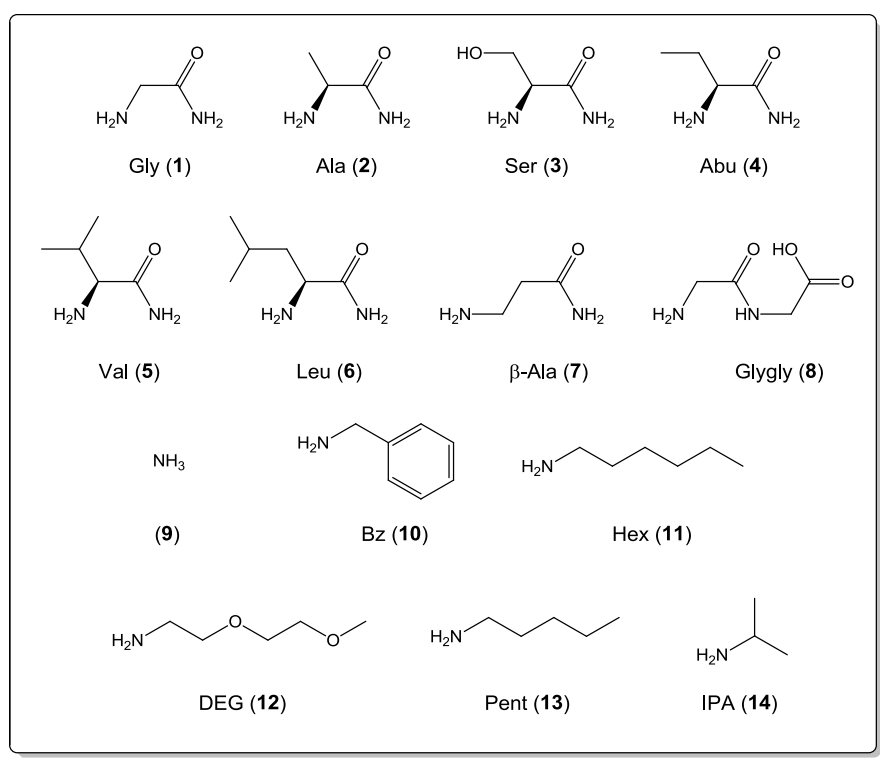

Scheme 1. Synthesis of homo- and copolymers from pPFPA with amines and structures of amines including abbreviations of analogous amino acids for amino acid amides 
Table 1. Overview of (Co)Polymers prepared from pPFPA with Feed Ratio of Amines, their Molar Composition determined by NMR, Molar Mass Details and Solubility Behavior.

\begin{tabular}{|c|c|c|c|c|c|c|c|c|}
\hline Entry & Name, Abbreviation & precursor & $\begin{array}{l}\text { Amine } \\
\text { A } \\
\text { (feed } \\
\text { ratio } \\
\text { in \%) }\end{array}$ & $\begin{array}{l}\text { Amine } \\
\text { B } \\
\text { (feed } \\
\text { ratio } \\
\text { in \%) }\end{array}$ & $\begin{array}{l}\mathrm{Mn}_{\mathrm{n}, \text { theor. }}{ }^{\mathrm{a}} \\
{[\mathrm{kg} / \mathrm{mol}]}\end{array}$ & $\begin{array}{l}\mathrm{M}_{\mathrm{n}, \mathrm{SEC}} \mathrm{b}^{\mathrm{b}} \\
{[\mathrm{kg} / \mathrm{mol}]}\end{array}$ & $\boldsymbol{\Phi}_{\mathrm{M}}^{\mathrm{b}}$ & $\begin{array}{l}\text { Behavior } \\
\left(\mathrm{CP} \text { in }{ }^{\circ} \mathrm{C}\right)\end{array}$ \\
\hline 1 & $\begin{array}{l}\text { Poly(N-acryloyl glycinamide), } \\
\text { pGlyAm }\end{array}$ & pPFPA1 & $\begin{array}{l}1 \\
(100 \%)\end{array}$ & & 13.4 & & & S \\
\hline 2 & $\begin{array}{l}\text { Poly( } N \text {-acryloyl alaninamide), } \\
\text { pAlaAm }\end{array}$ & pPFPA1 & $\begin{array}{l}2 \\
(100 \%)\end{array}$ & & 14.8 & 19.6 & 1.40 & I \\
\hline 3 & $\begin{array}{l}\text { Poly( } N \text {-acryloyl alaninamide), } \\
\text { pAlaAm }\end{array}$ & pPFPA2 & $\begin{array}{l}2 \\
(100 \%)\end{array}$ & & 5.8 & 8.9 & 1.41 & 1 \\
\hline 4 & $\begin{array}{l}\text { Poly }(N \text {-acryloyl serinamide), } \\
\text { pSerAm }\end{array}$ & pPFPA1 & $\begin{array}{l}3 \\
(100 \%)\end{array}$ & & 16.5 & 37.7 & 1.27 & $S$ \\
\hline 5 & $\begin{array}{l}\text { Poly(N-acryloyl aminobutyramide), } \\
\text { pAbuAm }\end{array}$ & pPFPA1 & $\begin{array}{l}4 \\
(100 \%)\end{array}$ & & 16.3 & 45.0 & 1.37 & S \\
\hline 6 & $\begin{array}{l}\text { Poly( } N \text {-acryloyl valinamide), } \\
\text { pValAm }\end{array}$ & pPFPA1 & $\begin{array}{l}5 \\
(100 \%)\end{array}$ & & 17.7 & 31.7 & 1.48 & 1 \\
\hline 7 & $\begin{array}{l}\text { Poly( } N \text {-acryloyl leucinamide), } \\
\text { pLeuAm }\end{array}$ & pPFPA1 & $\begin{array}{l}6 \\
(100 \%)\end{array}$ & & 19.1 & & & 1 \\
\hline 8 & $\begin{array}{l}\text { Poly }(N \text {-acryloyl } \beta \text {-alaninamide }), \\
\text { p( } \beta \text {-AlaAm) }\end{array}$ & pPFPA1 & 7 & & 14.8 & & & $S$ \\
\hline 9 & $\begin{array}{l}\text { Poly(N-acryloyl glycylglycine), } \\
\text { pGlyGlyAm }\end{array}$ & pPFPA1 & $\begin{array}{l}8 \\
(100 \%) \\
\end{array}$ & & 19.3 & & & $S$ \\
\hline 10 & $\begin{array}{l}\text { Poly }(N \text {-acryloyl glycinamide } 0.75-C o-N \\
\left.\text {-benzylacrylamide }{ }_{0.25}\right), p\left(\text { GlyAm }_{0.75-}\right. \\
\text { co-BzAmo.25) }\end{array}$ & pPFPA1 & $1(90 \%)$ & $\begin{array}{l}10 \\
(10 \%)\end{array}$ & 14.2 & 33.7 & 1.31 & $\mathrm{I}$ \\
\hline 11 & $\begin{array}{l}\text { Poly }\left(N \text {-acryloyl glycinamide }{ }_{0.70}-C o-N\right. \\
\left.\text {-hexylacrylamide }{ }_{0.30}\right), p\left(\text { GlyAm }_{0.70^{-}}\right. \\
\text {co-HexAm0.30) }\end{array}$ & pPFPA1 & $1(90 \%)$ & $\begin{array}{l}\mathbf{1 1} \\
(10 \%)\end{array}$ & 14.2 & 37.5 & 1.46 & 1 \\
\hline 12 & $\begin{array}{l}\text { Poly }(N \text {-acryloyl } \\
\text { aminobutyramide } 0.74^{-C o-N}- \\
\text { benzylacrylamide } \\
\left.\text { co-BzAm }{ }_{0.26}\right)\end{array}$ & pPFPA1 & $4(90 \%)$ & $\begin{array}{l}10 \\
(10 \%)\end{array}$ & 16.4 & 44.6 & 1.31 & $\begin{array}{l}\text { LCST } \\
(30.2)\end{array}$ \\
\hline 13 & $\begin{array}{l}\text { Poly }(N \text {-acryloyl } \\
\text { aminobutyramide }{ }_{0.76} \text {-Co- } N \text {-acryloyl } \\
\left.\text { leucinamide }_{0.24}\right), \mathrm{p}\left(\mathrm{AbuAm}_{0.76}-\mathrm{Co}-\right. \\
\left.\text { LeuAm }_{0.24}\right)\end{array}$ & pPFPA1 & $4(75 \%)$ & $6(25 \%)$ & 17.0 & 47.1 & 1.24 & $S$ \\
\hline 14 & $\begin{array}{l}\text { Poly }(N \text {-acryloyl } \\
\text { aminobutyramide } 0.55-C o-N \text {-acryloyl } \\
\left.\text { leucinamide }_{0.45}\right), \mathrm{p}\left(\text { AbuAm }_{0.55}-\mathrm{CO}^{-}\right. \\
\text {LeuAmo.45) }\end{array}$ & pPFPA1 & $4(50 \%)$ & $6(50 \%)$ & 17.6 & 40.2 & 1.48 & $\begin{array}{l}\text { LCST } \\
(43.8)\end{array}$ \\
\hline 15 & $\begin{array}{l}\text { Poly( } N \text {-acryloyl } \\
\text { aminobutyramide }{ }_{0.29-c o-N} \text {-acryloyl } \\
\left.\text { leucinamide }_{0.71}\right), \mathrm{p}(\text { AbuAmo.29-Co- } \\
\left.\text { LeuAm }_{0.71}\right)\end{array}$ & pPFPA1 & $4(25 \%)$ & $6(75 \%)$ & 18.3 & 43.5 & 1.40 & 1 \\
\hline 16 & 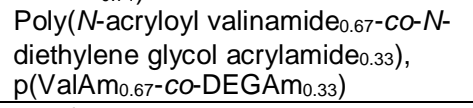 & pPFPA1 & $5(90 \%)$ & $\begin{array}{l}12 \\
(10 \%)\end{array}$ & 17.8 & & & $\begin{array}{l}\text { LCST } \\
(31.8)\end{array}$ \\
\hline 17 & $\begin{array}{l}\text { Poly }(N \text {-acryloyl alaninamide } 0.73-C O- \\
N \text { - pentylacrylamide } 0.27) \\
\text { p(AlaAm } \\
\left.0.73-C o-\text { Pent } A m_{0.27}\right)\end{array}$ & pPFPA2 & $2(80 \%)$ & $\begin{array}{l}13 \\
(20 \%)\end{array}$ & 5.8 & 10.3 & 1.27 & I \\
\hline 18 & $\begin{array}{l}\text { Poly }(N \text {-acryloyl alaninamide } 0.78-c o- \\
N \text {-diethylene glycol acrylamide } \\
\text { p(AlaAmo.22), }\end{array}$ & pPFPA2 & $2(80 \%)$ & $\begin{array}{l}12 \\
(20 \%)\end{array}$ & 6.0 & 11.1 & 1.27 & $S$ \\
\hline 19 & $\begin{array}{l}\text { Poly }(N \text {-acryloyl alaninamide } \\
N \text {-acryloyl glycinamide }{ }_{0.54} \text {, } \\
\text { p(AlaAmo.46-Co-GlyAmo.54) }\end{array}$ & pPFPA1 & $2(50 \%)$ & $1(50 \%)$ & 14.0 & & & $S$ \\
\hline 20 & $\begin{array}{l}\text { Poly(N-acryloyl alaninamide }{ }_{0.38}-c o- \\
N \text {-isopropylacrylamide } 0.62) \\
\text { p(AlaAmm }\end{array}$ & pPFPA1 & $2(20 \%)$ & $\begin{array}{l}14 \\
(80 \%)\end{array}$ & 13.0 & 62.1 & 1.29 & $\begin{array}{l}\text { LCST } \\
(50.9)\end{array}$ \\
\hline 21 & Poly(acrylamide), pAm & pPFPA1 & $\begin{array}{l}\mathbf{9} \\
(100 \%)\end{array}$ & & 7.5 & & & $\mathrm{~S}$ \\
\hline 22 & $\begin{array}{l}\text { Poly(acrylamide }{ }_{0.94}-\mathrm{co}-\mathrm{N}- \\
\left.\text { benzylacrylamide }_{0.06}\right), \mathrm{p}\left(\mathrm{Am}_{0.94-}-\mathrm{Co}-\right. \\
\left.\text { BzAm }_{0.06}\right)\end{array}$ & pPFPA1 & 9 (95\%) & $10(5 \%)$ & 8.1 & & & S \\
\hline 23 & $\begin{array}{l}\text { Poly(acrylamide }{ }_{0.87}-\mathrm{co}-\mathrm{N}- \\
\left.\text { benzylacrylamide }_{0.13}\right), \mathrm{p}\left(\mathrm{Am}_{0.87-\mathrm{co}-}\right. \\
\left.\text { BzAm }_{0.13}\right)\end{array}$ & pPFPA1 & $9(90 \%)$ & $\begin{array}{l}10 \\
(10 \%)\end{array}$ & 8.7 & & & UCST (7.1) \\
\hline 24 & $\begin{array}{l}\text { Poly(acrylamide } 0.81-c o-N- \\
\left.\text { benzylacrylamide }_{0.19}\right), p\left(\text { Am }_{0.81}-c o-\right. \\
\left.\text { BzAm }_{0.19}\right)\end{array}$ & pPFPA1 & 9 (85\%) & $\begin{array}{l}10 \\
(15 \%)\end{array}$ & 9.3 & & & I \\
\hline 25 & $\begin{array}{l}\text { Poly(acrylamide }{ }_{0.69}-c o-N- \\
\left.\text { benzylacrylamide }{ }_{0.31}\right), p\left(\mathrm{Am}_{0.69-c o-}\right. \\
\text { BzAmmo.31 }\end{array}$ & pPFPA1 & $9(80 \%)$ & $\begin{array}{l}10 \\
(20 \%)\end{array}$ & 10.4 & & & I \\
\hline
\end{tabular}


${ }^{\text {a }}$ Molar mass of (co)polymers calculated from degree of polymerization of precursor and copolymer composition from NMR

${ }^{\mathrm{b}}$ Polystyrene equivalent molecular weight and dispersity $\left(\bigoplus_{\mathrm{M}}\right)$ determined by SEC.

${ }^{\mathrm{c}}$ Solubility in water (concentration $5 \mathrm{~g} / \mathrm{L}, 0-90^{\circ} \mathrm{C}$ ) $\mathrm{S}=$ soluble, $\mathrm{I}=$ insoluble; $\mathrm{CP}=$ cloud point determined at $50 \%$ transmittance
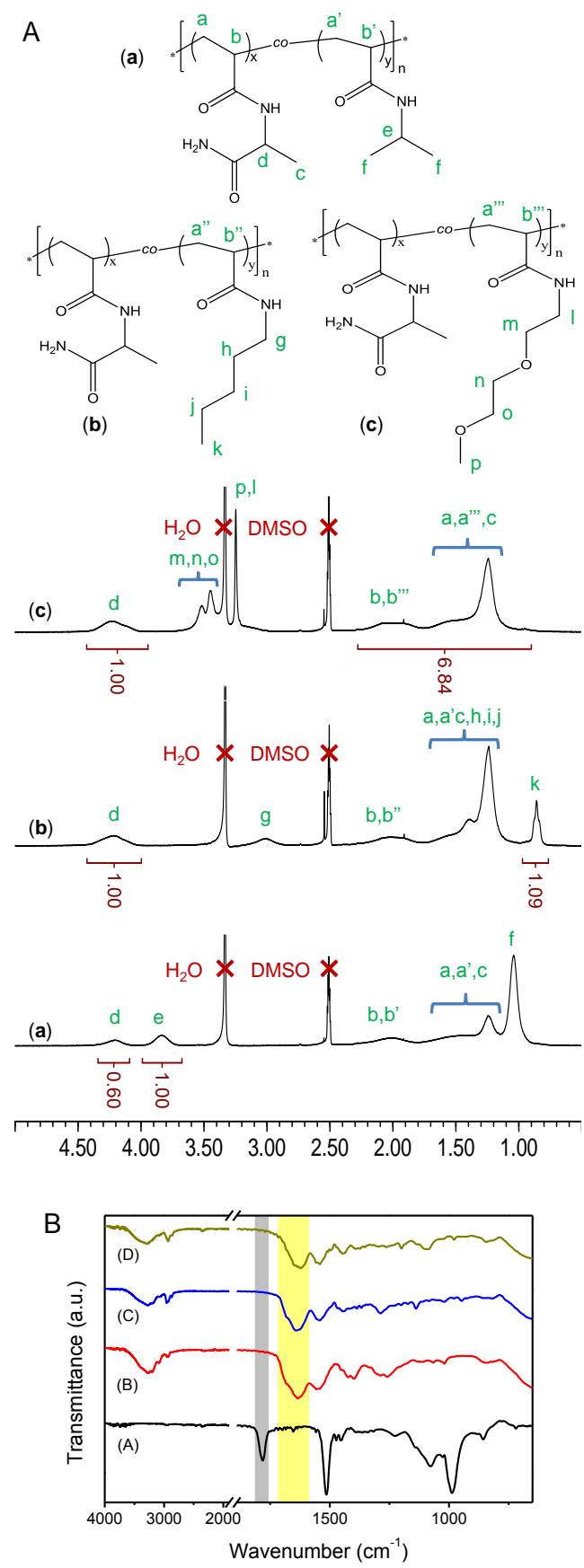

Figure 1. A) ${ }^{1} \mathrm{H}$ NMR spectra measured in DMSO- $\mathrm{d}_{6}$ of the Ala-containing copolymers p(AlaAm0.38-co-IPAm 0.62 ) (a); p(AlaAm0.73-co-PentAm 0.27 ) (b); and p(AlaAm0.78-coDEGAm $_{0.22}$ ) (c) with peak assignments. B) FT-IR spectra of the pPFPA precursor (A), pGlyAm (B), pLeuAm (C), and p(ValAm $\left.{ }_{0.67-c o-D E G A m}{ }_{0.33}\right)$ (D). The characteristic bands of the activated ester $\mathrm{C}=\mathrm{O}$ stretch (gray) and the amide $\mathrm{C}=\mathrm{O}$ stretch (yellow) are marked. 


\section{Aqueous Solution Behavior}

With a library of novel (bis)amide (co)polymers prepared by postpolymerization modification of pPFPA in hand, we next investigated their aqueous solution behavior. pGlyAm (Table 1, entry 1) exhibited thermoreversible gelation in water at concentrations above $15 \mathrm{wt} \%$ (Figure $2 \mathrm{AB})$, reversibly switching between a gelled and a free-flowing state, as described previously for this species,[2] and confirming successful synthesis of a thermoresponsive species through postmodification of pPFPA. The gel-sol transition of pGlyAm at a concentration of $30 \mathrm{wt} \%$ (photograph in Figure $2 \mathrm{AB}$ ) occurred at $75^{\circ} \mathrm{C}$; gelation upon cooling occurred at $69^{\circ} \mathrm{C}$, exhibiting a hysteresis of $6{ }^{\circ} \mathrm{C}$. Two further (co)polymer samples, p(AlaAm $0.46-\mathrm{Co}$ GlyAm$_{0.54}$ ) (entry 19) and pSerAm (entry 4), at concentrations above $30 \mathrm{wt} \%$, also formed gels in water which melted upon heating $\left(79^{\circ} \mathrm{C}\right.$ for $\mathrm{p}\left(\mathrm{AlaAm}_{0.46}-\mathrm{co}-\mathrm{GlyAm}_{0.54}\right), 80{ }^{\circ} \mathrm{C}$ for pSerAm) forming a more viscous, 'honey-like' liquid. Upon cooling, gelation occurred with a hysteresis of $8{ }^{\circ} \mathrm{C}$ indicating thermoreversible behavior.

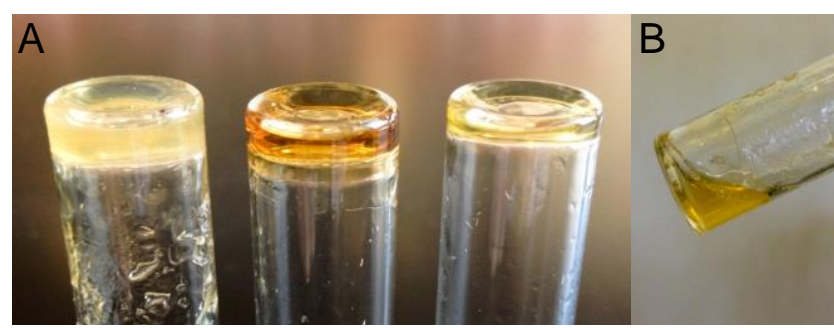

Figure 2. A) Photographs of (left to right) pGlyAm, pSerAm, and p(AlaAm ${ }_{0.46}-\mathrm{co}-$ GlyAm 0.54 ) gels in water (30 wt-\%); B) Photograph of same pGlyAm sample after heating. The yellow color is due to RAFT agent end groups.

In order to identify species with thermoresponsive behavior in more dilute solution, (co)polymers were tested for water solubility at a concentration of $5 \mathrm{~g} / \mathrm{L}$ in the temperature range $0-90{ }^{\circ} \mathrm{C}$; results are summarized in Table 1 . Within the series of homopolymers (Table 1, entries 1-9) pGlyAm, p( $\beta$-AlaAm), pAbuAm (formally pGlyAm with pendant ethyl 
chains), and pGlyGlyAm were water soluble while the more hydrophobic species pValAm and pLeuAm (isopropyl and isobutyl side chains) were insoluble. pGlyAm did not show UCST behavior, possibly due to acrylic acid contamination of the monomer or polymer through hydrolysis reactions.[10] Surprisingly, pAlaAm (entry 2, methyl side chains) was found to be insoluble, suggesting stronger inter- and intramolecular hydrogen bonding than in the Abu analogue and the isomeric $\beta$-Ala species. Insolubility of pAlaAm was also confirmed on a sample with a lower molecular weight $\left(M_{\mathrm{n}}=5.8 \mathrm{~kg} / \mathrm{mol}\right.$, entry 3) prepared from pPFPA2. With amino-acid based bisamide polymers receiving attention as (UCST-type) thermoresponsive materials, we investigated the possibility of realizing LCST transitions within this family of polymers by tuning their hydrophilic-hydrophobic balance,[35] which is easily achieved by postpolymerisation modification by employing a mixture of modifying reagents.[20, 21] First, we hydrophobically modified the water soluble species pGlyAm and pAbuAm. pGlyAm-based copolymers containing $25 \mathrm{~mol} \%$ benzylacrylamide and $30 \mathrm{~mol} \%$ hexylacrylamide (entries 10 and 11) were both insoluble in water. Containing 26 mol\%

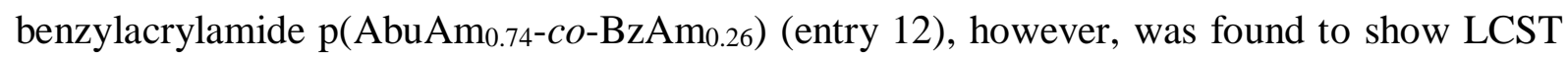
behavior in water with a measured cloud point of $30^{\circ} \mathrm{C}$. In a series of copolymers of AbuAm and LeuAm, entries 13-15, p(AbuAm 0.76 -co-LeuAm 0.24$) \quad$ (soluble), p(AbuAm 0.55 -coLeuAm $_{0.45}$ ) (LCST cloud point at $\left.44{ }^{\circ} \mathrm{C}\right)$, and $\mathrm{p}\left(\mathrm{AbuAm}_{0.29}\right.$-co-LeuAmo.71) (insoluble) solubility decreased-expectedly—with increasing LeuAm content, showing successful design of an LCST-type copolymer consisting entirely of amino acid amide-derived monomer units. These observations confirm that (ethyl side chain) AbuAm segments are indeed hydrophilic (in spite of the methyl side chain analogous polymer pAlaAm being insoluble), since their incorporation into the hydrophobic pLeuAm species increased solubility and evoked LCST behavior. Of note, both AbuAm-based copolymers p(AbuAm0.55-coLeuAm $\left._{0.45}\right)$ and $\mathrm{p}\left(\mathrm{AbuAm}_{\left.0.74-\mathrm{co}-\mathrm{BzAm}_{0.26}\right)}\right.$ showed broad LCST transitions with transmittance decreasing gradually over a range of $15-20{ }^{\circ} \mathrm{C}$, Figure $3 \mathrm{~A}$. LCST behavior 
could also be induced by means of hydrophilic modification of the water insoluble species pValAm. Containing $33 \mathrm{~mol} \%$ of DEG acrylamide comonomer, p(ValAm $0.67-\mathrm{Co}^{-}$ DEGAm 0.33 ), entry 16 , showed a relatively sharp cloud point at $32{ }^{\circ} \mathrm{C}$, Figure $3 \mathrm{~A}$, suggesting that, irrespective of the bisamide functionality, tuning of aqueous solution behavior and realization of LCST transitions was possible. We next investigated pAlaAm-based polymers in more detail due to the unexpected insolubility of the pAlaAm homopolymer in water and prepared four copolymers, Table 1 entries 17-20. Their aqueous solution behavior appeared to be dictated by the nature of the comonomer. Containing $24 \mathrm{~mol} \%$ pentylacrylamide, $\mathrm{p}\left(\right.$ AlaAm $_{0.73}$-co-PentAm 0.27$)$ was insoluble, while incorporation of $18 \mathrm{~mol} \%$ DEG acrylamide comonomer resulted in full aqueous solubility. Likewise, a near-equimolar copolymer of Ala and Gly-based segments was soluble in water. Reaction of pPFPA with a mixture of isopropyl amine and alaninamide produced a pNIPAM-based copolymer containing $38 \mathrm{~mol} \%$ Ala-units. This copolymer exhibited LCST behavior with an onset of transmittance decrease around $32{ }^{\circ} \mathrm{C}$, the well-documented LCST of pNIPAM,[36] though with an extremely broad transition, apparently caused by the presence of the strong hydrogen bonding comonomer units. While (co)polymers exhibiting sharp thermal transitions are often considered ideal systems, we note that gradual transitions (for example those found for UCST transitions in water-alcohol mixtures) have specifically been exploited for sensing applications over a wider temperature range than would be possible with typical LCST systems.[37]

To the best of our knowledge, thermoresponsive (co)polymers derived through postmodification of activated ester precursors have only exhibited LCST-type transitions. Intending to also realize UCST transitions employing this method, we prepared a series of acrylamide-benzylacrylamide copolymers, Table 1 entries $21-25$. With low amounts (0 and $6 \mathrm{~mol} \%$ ) of the hydrophobic modifier, the resulting (co)polymers were soluble in water, while a sample containing 13 mol\% benzylacrylamide showed a gradual UCST transition in water 
(Figure 3B). With higher contents of benzylacrylamide, however, polymers could not be dissolved in water, suggesting poor control over UCST tuning using this synthetic approach.
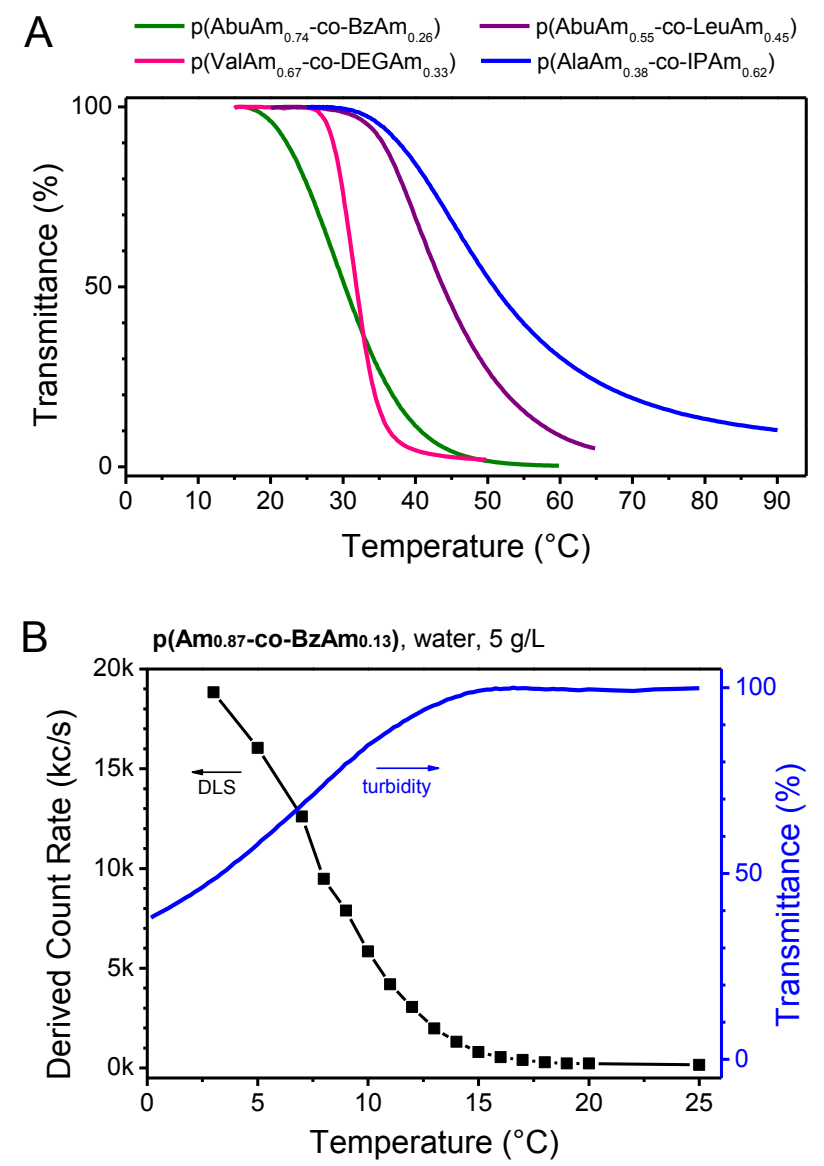

Figure 3. A) Transmittance curves of heating copolymer solutions showing LCST behavior in water (cooling curves shown in supporting information) B) Scattering intensity (by DLS, black curve) and transmittance (by turbidity, blue curve $)$ of $\mathrm{p}\left(\mathrm{Am}_{0.87}-\mathrm{co}\right.$-BzAmo.13 $)$ showing concurrent decrease of transmittance and increase of scattering count rate upon cooling indicating UCST behavior in water. 


\section{Self-Association}

Apart from effecting thermoreversible attractions between polymer chains during gelation or UCST transitions in certain (co)polymer species, the strong hydrogen bonding capacity of amide groups plays an integral role in directed self-association, for example in the folding of proteins into quaternary structures or the helical structure of DNA. Inspired by nature, such attractions have been widely exploited in the supramolecular arena for the generation of 1D, 2D, and 3D highly ordered and hierarchical assemblies.[38, 39] While hydrogen bonding polymer-polymer attractions in aqueous solution of most (co)polymer species presented here were, apparently, not sufficient to evoke UCST transitions, we explored their ability to direct self-assembly into ordered structures in the dry state. Aqueous (co)polymer solutions (concentration $2 \mathrm{~g} / \mathrm{L}$ ) were freeze-dried and the morphology of the resulting solids was analyzed by scanning electron microscopy (SEM) after coating with a thin layer of chromium, Figure 4. Uniform, comparably large structures (tens to hundreds of micrometers in size), which differed dramatically with the chemical composition of the (co)polymers were observed. For example, pGlyAm self-associated into rod-like structures with diameters of 350-450 $\mathrm{nm}$ and lengths in excess of $35 \mu \mathrm{m}$ (Figure $4 \mathrm{a}, \mathrm{b}$ ). In contrast to pGlyAm, $\mathrm{p}\left(\mathrm{AlaAm}_{0.46}\right.$-co-GlyAm 0.54$)$-formally pGlyAm with 46 mol\% stereoregular methylsubstitution — formed stacked sheets with edge lengths $>100 \mu \mathrm{m}$ with worm-like outgrowths supporting the sheets at a distance of approximately $4.5 \mu \mathrm{m}$ (Figure $4 \mathrm{c}, \mathrm{d}$ ). A sheet-like morphology, though without worm-like outgrowths and an order of magnitude larger in size, with sheet lengths of several hundred micrometers, and sheet thicknesses of $\sim 250 \mathrm{~nm}$ was also observed for pGlyGlyAm, see Figure $4 \mathrm{~g}$,h. The LCST sample p(AbuAm0.74-co-BzAm 0.26 , on the other hand, self-associated into honeycomb-like structures with rectangular pores of $\sim 2.5 \times 15 \mu \mathrm{m}$ sizes and wall thicknesses of $\sim 200 \mathrm{~nm}$, Figure 4 e,f. Such order and uniformity of observed structures is impressive, as is the strong influence of chemical structure on the observed morphology. Of note, a sample of the pNIPAM-based species $\mathrm{p}\left(\mathrm{AlaAm}_{0.38^{-}-\mathrm{co}-}\right.$ 
IPAm $_{0.62}$ did not assemble into uniform structures giving a mixture of sheets, rods, and spherical structures, suggesting a possible importance of bisamide moieties and, perhaps, stereoregularity throughout the polymer, see supporting information. Vesicular morphology of end-group modified polymers after freeze-drying polymer solutions has previously been described,[40, 41] though this process is not fully understood. We assume that structuring occurs during freeze-drying at the air-ice interface where a gradual evaporation of their hydration sphere allows (co)polymer chains a certain mobility (that may well be governed by the manner in which individual water molecules are removed from the backbone and the two amide side groups) which causes chains to self-associate in a particular fashion, affording a growth of regular structures. The direction in which the ice evaporated from the sample could give rise to the anisotropic character of the observed structures. With large interest in producing self-assembled regular structures, we believe that our observations offer great potential for applications requiring large surface areas, e.g. catalysis, or in the fabrication of membranes. 


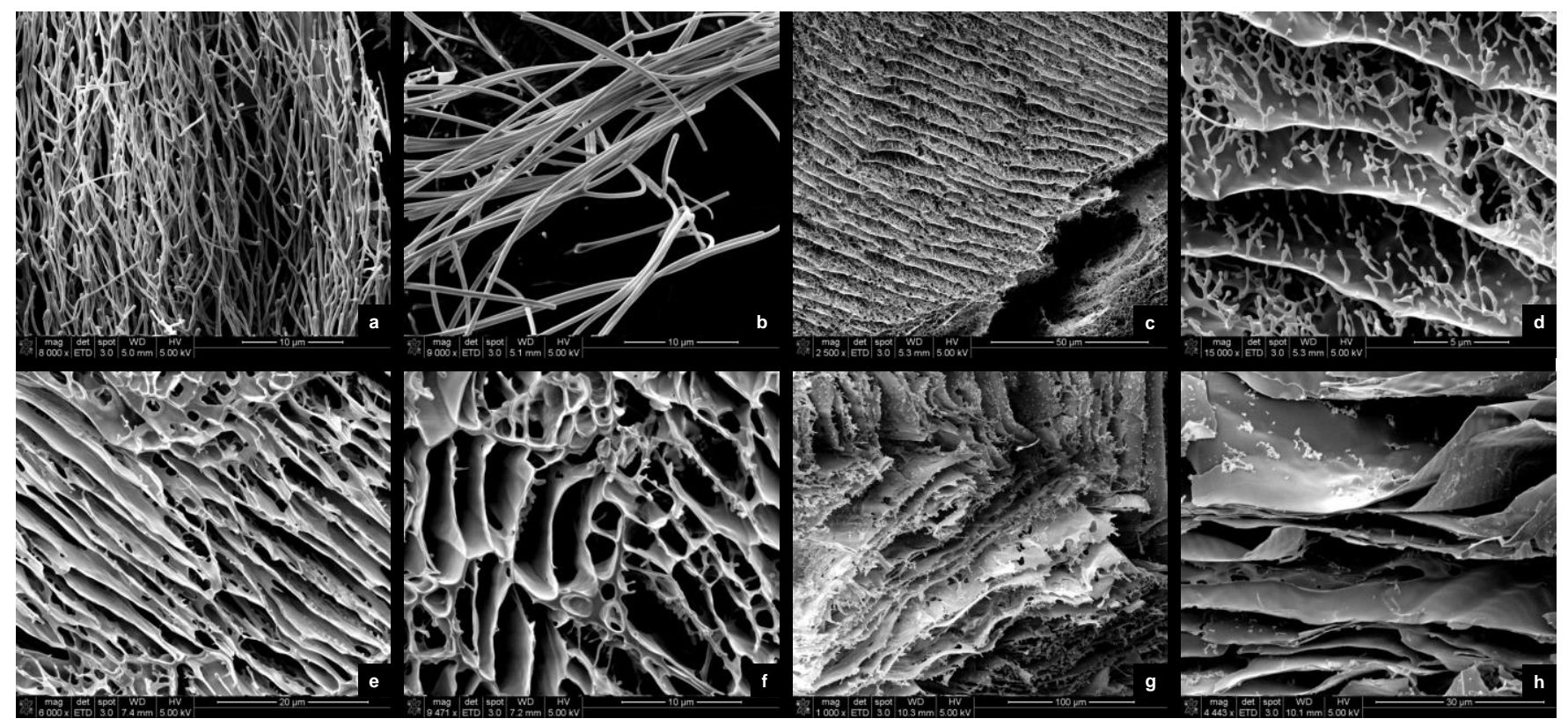

Figure 4. SEM images of dry pGlyAm (a, b; scale bars $10 \mu \mathrm{m}), \mathrm{p}\left(\mathrm{AlaAm}_{\left.0.46-c o-\text { GlyAm }_{0.54}\right)}\right.$ (c, d; s.b. $50 \mu \mathrm{m}$ and $5 \mu \mathrm{m})$, p(AbuAm0.74-co-BzAm 0.26 ) (e, f; s.b. $20 \mu \mathrm{m}$ and $10 \mu \mathrm{m})$, and pGlyGlyAm $(\mathbf{g}, \mathbf{h} ; 100 \mu \mathrm{m}$ and $30 \mu \mathrm{m})$

\section{Conclusions}

Postpolymerization modification of activated ester precursors provided access to a library of novel, well-defined bisamide (co)polymers and hydrophobically modified acrylamide copolymers. While UCST behavior, as described in the literature for several of such polymeric materials, was only found for one sample, poly(acrylamide $0.87-c o-N$ benzylacrylamide 0.13 ), several bisamide copolymers were designed to show LCST behavior exhibiting very broad transitions. Interestingly, (co)polymers self-associated into highly regular, ordered structures upon freeze-drying, a promising phenomenon that we are currently investigating in more detail. 
Acknowledgements: P.J.R. acknowledges the Australian Research Council (ARC) for funding through a Discovery Early Career Researcher Award (DE120101547).

Keywords: phase behavior, self-organization, gelation, thermoresponsive, postpolymerization modification 


\section{Graphical Abstract}

Yicheng Zhu, Andrew B. Lowe, Peter J. Roth*

Postpolymerization Synthesis of (Bis)Amide (Co)Polymers: Thermoresponsive Behavior and Self-association

Governed by strong intra- and intermolecular hydrogen

bonding between amide groups, a series of novel

(bis)amide (co)polymers prepared by post-polymerization modification of an activated ester scaffold is shown to reversibly gel in water at low temperatures, display LCST or UCST behavior in water and to self-organize into large

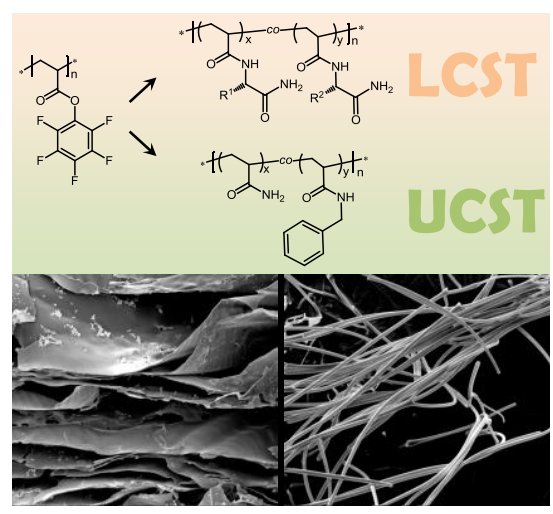
sheet-like or rod-like structures. 
1. Haas HC, Moreau RD, and Schuler NW. Journal of Polymer Science Part A-2:

Polymer Physics 1967;5(5):915-927.

2. Glatzel S, Badi N, Päch M, Laschewsky A, and Lutz J-F. Chemical Communications 2010;46(25):4517-4519.

3. Eguchi M and Ohnishi N. EP 2009044 A1 2008;December 31, 2008.

4. Nagaoka H, Ohnishi N, and Eguchi M. US Pat. 7847047 B2, Chisso Corporation, Osaka (JP) 2010.

5. Ohnishi N, Furukawa H, Kataoka K, and Katsuhiko U. US Pat. 7195925 B2, National Institute of Advanced Industrial Science and Technology, Chisso Corporation 2007.

6. Glatzel S, Laschewsky A, and Lutz J-F. Macromolecules 2011;44:413-415.

7. Seuring J and Agarwal S. Macromolecular Chemistry and Physics 2010;211(19):21092117.

8. Seuring J and Agarwal S. Macromol Rapid Commun 2012;33(22):1898-1920.

9. Seuring J and Agarwal S. ACS Macro Letters 2013;2(7):597-600.

10. Seuring J, Bayer FM, Huber K, and Agarwal S. Macromolecules 2012;45(1):374-384.

11. Liu F, Seuring J, and Agarwal S. Journal of Polymer Science Part A: Polymer Chemistry 2012;50(23):4920-4928.

12. Liu F, Seuring J, and Agarwal S. Polymer Chemistry 2013;4(10):3123-3131.

13. Seuring J and Agarwal S. Macromolecules 2012;45(9):3910-3918.

14. Günay KA, Theato P, and Klok H-A. Journal of Polymer Science Part A: Polymer Chemistry 2013;51(1):1-28.

15. Gauthier MA, Gibson MI, and Klok H-A. Angewandte Chemie International Edition 2009;48(1):48-58.

16. Günay KA, Theato P, and Klok H-A. History of Post-Polymerization Modification. Functional Polymers by Post-Polymerization Modification: Wiley-VCH Verlag GmbH \& Co. KGaA, 2012. pp. 1-44. 
17. Roth PJ, Wiss KT, and Theato P. 5.12 - Post-Polymerization Modification. In:

Matyjaszewski K and Möller M, editors. Polymer Science: A Comprehensive Reference.

Amsterdam: Elsevier, 2012. pp. 247-267.

18. Theato P. Journal of Polymer Science Part A: Polymer Chemistry 2008;46(20):66776687.

19. Roth PJ. Macromol. Chem. Phys. 2014:DOI 10.1002/macp.201400073.

20. Zhu Y, Quek JY, Lowe AB, and Roth PJ. Macromolecules 2013;46(16):6475-6484.

21. Chua GBH, Roth PJ, Duong HTT, Davis TP, and Lowe AB. Macromolecules 2012;45(3):1362-1374.

22. Jochum FD, Roth PJ, Kessler D, and Theato P. Biomacromolecules 2010;11(9):24322439.

23. Jochum FD and Theato P. Macromolecules 2009;42(16):5941-5945.

24. Jochum FD and Theato P. Polymer 2009;50(14):3079-3085.

25. Quek JY, Roth PJ, Evans RA, Davis TP, and Lowe AB. Journal of Polymer Science Part A: Polymer Chemistry 2013;51(2):394-404.

26. Quek JY, Zhu Y, Roth PJ, Davis TP, and Lowe AB. Macromolecules 2013;46(18):7290-7302.

27. Schattling P, Jochum FD, and Theato P. Chemical Communications 2011;47(31):8859-8861.

28. Schattling P, Pollmann I, and Theato P. Reactive and Functional Polymers 2014;75(0):16-21.

29. Mishra V, Jung S-H, Jeong HM, and Lee H-i. Polymer Chemistry 2014;5(7):2411.

30. Shimada N, Nakayama M, Kano A, and Maruyama A. Biomacromolecules 2013;14(5):1452-1457.

31. Woodfield PA, Zhu YC, Pei YW, and Roth PJ. Macromolecules 2014;47(2):750-762. 
32. Moad G, Rizzardo E, and Thang SH. Australian Journal of Chemistry 2012;65(8):9851076.

33. Harvison MA, Roth PJ, Davis TP, and Lowe AB. Aust. J. Chem 2011;64:992-1006.

34. Roth PJ, Boyer C, Lowe AB, and Davis TP. Macromolecular Rapid Communications 2011;32(15):1123-1143.

35. Taylor LD and Cerankowski LD. Journal of Polymer Science: Polymer Chemistry Edition 1975;13(11):2551-2570.

36. Schild HG. Progress in Polymer Science 1992;17(2):163-249.

37. Pietsch C, Hoogenboom R, and Schubert US. Polymer Chemistry 2010;1(7):10051008.

38. Chapman R, Danial M, Koh ML, Jolliffe KA, and Perrier S. Chemical Society Reviews 2012;41(18):6023-6041.

39. Stupp SI and Palmer LC. Chemistry of Materials 2013;26(1):507-518.

40. Roth PJ, Davis TP, and Lowe AB. Macromol Rapid Commun 2014;35(8):813-820.

41. Xu J, Tao L, Boyer C, Lowe AB, and Davis TP. Macromolecules 2010;44(2):299-312. 